\title{
LE TRAITEMENT HYDROTHERMAL DES DÉCHETS : UNE SOLUTION INNOVANTE
}

Patrick Beslin*, François Cansell*, Yves Garrabos*, Gérard Demazeau*, Bernard Berdeu**, Dominique Sentagnes** ICMCB-CNRS, L'Électrolyse

Nos sociétés industrialisées génèrent des volumes de déchets, notamment des déchets ultimes, dont les coûts de traitement ou de stockage dans les centres d'enfouissements techniques ne cessent de croître. Face à cette problématique, l'oxydation hydrothermale des déchets a été développée pour limiter notamment le volume des déchets ultimes. Dans ce cadre, nous avons développé dans notre laboratoire, en collaboration avec une PME bordelaise, l'Électrolyse, un pilote traitant $3 \mathrm{~kg} / \mathrm{h}$ en continu d'effluents liquides chargés à $10 \%$ en poids de déchets organiques.

Après une présentation de l'évolution des propriétés thermophysiques de l'eau, nous décrirons brièvement les principaux procédés commerciaux, pilotes ou concepts qui sont développés dans les domaines du traitement de déchets en milieux souscritique et supercritique. Nous présenterons ensuite le pilote de traitement de déchets que nous avons développé à l'ICMCB et les résultats obtenus concernant le traitement de deux molécules modèles : le méthanol et le glucose. L'influence des paramètres opératoires sur les taux de transformations des effluents traités seront discutés (pression, température, temps de séjour, additifs). Les conditions opératoires optimales ont été définies et ont permis d'obtenir des taux de destruction supérieur à $99 \%$. Enfin nous discuterons les aspects cinétiques mis en jeu dans ce type de réactions.

\section{INTRODUCTION}

Nos sociétés industrialisées génèrent des volumes de déchets dont les coûts de traitement ou de stockage dans les centres d'enfouissements techniques (CET) ne cessent de croître. A titre d'exemple, la France a produit en 1994, 700 à 800 mille tonnes de boues issues du traitement des eaux de collectivités locales et I à I,2 million de tonnes de boues industrielles. Aujourd'hui, il n'est plus suffisant, dans le cas du traitement de l'eau, de faire un transfert de pollution d'un milieu dilué à un milieu concentré que l'on va stocker dans un CET. En effet, au $I^{\text {er }}$ juillet 2002, seuls les déchets ultimes inertés (non valorisables, stables, non toxiques, non fermentescibles,...) pourront être mis en décharge. Face à cette contrainte, un effort important de recherche et développement a été entrepris pour la mise au point de nouvelles
The environmental regulation evolution and the increasing wastewater disposal cost lead to a new concept of complete destruction of toxic substances and sludges. Thus, hydrothermal oxidation of wastes as an alternative technique in order to limit the risks of secondary pollution is developed. In this field, we have developed in our laboratory, in collaboration with the Electrolyse (Bordeaux - France), a continuous reactor which permits to treat $3 \mathrm{~kg}$ per hour of wastes containing 10 weight percent of organic compounds.

After a brief presentation of supercritical water properties and technological developments of hydrothermal oxidation of wastes, we will present the results obtained in our laboratory concerning two model molecules : methanol and glucose. The influence of various parameters governing the transformation has been investigated (pressure, temperature, residence time, additive). The kinetics has been modeled for elementary compounds. This study has evidenced high destruction rates (>99\%).

technologies (torche à plasma, pyrolyse, traitement hydrothermal,...). A court terme, les objectifs principaux sont : - l'absence d'émission secondaire polluante en phase gaz ; - la valorisation des déchets ;

- la réduction du volume ultime des déchets ;

- l'inertage des déchets ultimes.

Afin de répondre à ces objectifs, un travail important de recherche fondamentale sur l'oxydation hydrothermale a été initié aux États-Unis, puis en Europe et au Japon. II s'est traduit par la parution d'une littérature scientifique abondante et par le dépôt de nombreux brevets. Après avoir présenté l'évolution des propriétés thermophysiques de l'eau lorsque l'on se déplace dans l'espace des phases autour du point critique (coordonnées critiques de l'eau : $\mathrm{Tc}=374^{\circ} \mathrm{C}$, $P c=22,1 \mathrm{MPa}$ et $\rho c=322 \mathrm{~kg} / \mathrm{m}^{3}$ ), nous décrirons briève- 
ment les principaux procédés commerciaux, pilotes ou concepts qui sont développés dans les domaines du traitement de déchets en milieux souscritique et supercritique. Le principe général commun à ces deux domaines de pression et de température est de transformer une charge organique en sous produits gazeux non toxiques. Dans le cas de l'oxydation, si la réaction est totale, les produits en sortie de réacteur sont constitués de $\mathrm{CO}_{2}$ et d' $\mathrm{H}_{2} \mathrm{O}$. Nous présenterons ensuite le pilote de traitement de déchets que nous avons développé à l'ICMCB et les résultats obtenus concernant le traitement de deux molécules modèles : le méthanol et le glucose.

\section{PROPRIÉTÉS THERMOPHYSIQUES DE L'EAU PURE}

La figure I présente le diagramme de phase de l'eau pure ${ }^{[1]}$ et l'évolution de sa masse volumique qui peut être modulée de manière continue de l'état gazeux à l'état liquide pour de faibles variations des paramètres pression et température en contournant le point critique liquide-gaz. La polarité de l'eau peut, elle aussi, être contrôlée par ces deux paramètres. Elle augmente avec la pression et diminue fortement avec la température. A titre d'exemple, la constante diélectrique de l'eau à $25 \mathrm{MPa}$ chute d'une valeur de 80 environ à température ambiante à une valeur de 2 à $450^{\circ} \mathrm{C}^{[2]}$. Dans des conditions de pression et de température supercritiques, l'eau devient un solvant peu polaire ce qui conduit à une diminution importante de la solubilité des sels inorganiques. Par contre, la solubilité des composés organiques croît jusqu'à leur miscibilité totale, notamment pour le benzène.

L'ajout d'autres composants dans l'eau pure induit une modification du diagramme de phase, et les coordonnées critiques peuvent être déplacées dans des proportions parfois non négligeables. E.-U. Franck a observé un déplacement significatif vers les hautes températures du diagramme de phase binaire eau/méthane avec l'ajout de faibles quantités de chlorure de sodium ${ }^{[3]}$.

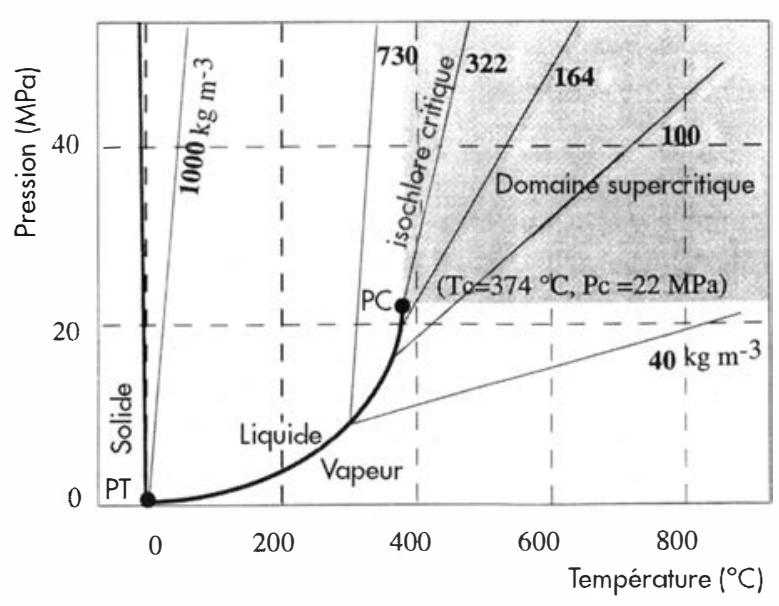

Figure I : Diagramme de phase de l'eau pure

\section{DÉVELOPPEMENTS TECHNOLOGIQUES}

Le procédé d'oxydation en milieu souscritique $\left(150^{\circ} \mathrm{C}<\mathrm{T}\right.$ $<325^{\circ} \mathrm{C}, 2 \mathrm{MPa}<\mathrm{P}<20 \mathrm{MPa}$ ) a été commercialisé aux États-Unis à partir de 1958 (procédé Zimpro). Ce type de procédé a démontré son efficacité pour la régénération de charbon actif et dans le traitement de nombreux déchets aqueux tels que : des boues de stations d'épuration; des boues de papeterie ; des cyanures organiques ou minéraux ; des sulfures ; des hydrocarbures aliphatiques; des phénols ; des aromatiques; des hétérocycles $(\mathrm{O}, \mathrm{N}, \mathrm{S})$. Dans de nombreuses applications, l'oxydation humide est utilisée en post traitement, afin de réduire suffisamment la concentration en composés toxiques pour permettre un traitement biologique classique $\mathrm{e}^{[4.7]}$. Les principales étapes du procédé peuvent être décrites comme suit : pressurisation du déchet aqueux et de l'additif (oxydant) ; préchauffage ; réaction ; refroidissement ; dépressurisation ; séparation gaz-liquide ; gestion des effluents. Les réacteurs peuvent se présenter sous quatre formes :

- réacteur en forme de colonne verticale (Zimpro/CibaGeigy) ${ }^{[8-10]}$;

- réacteur agité (Wetox-Zimpro). Cette technologie utilise un agitateur mécanique ;

- réacteur constitué de plusieurs tubes concentriques disposés dans un puits de forage (Vertech) ${ }^{[1]}$;

- réacteur batch pour le traitement de déchets hétérogènes $^{[12]}$.

Au niveau des laboratoires publics européens, un pilote continu de traitement d'effluents organiques et minéraux (18 I/heure) est opérationnel à l'université d'Erlangen (Allemagne). Les études portent essentiellement sur l'utilisation de catalyseurs et l'inhibition des phénomènes de corrosion $^{[13]}$.

La nature des effluents ainsi que le bilan énergétique de chacun de ces procédés ne peuvent être discutés à partir de considérations générales tant la nature de la charge à détruire conditionne les performances environnementales et économiques de cette technologie. Cependant, l'oxydation humide présente un intérêt pour les déchets dilués contenant des produits non réfractaires à l'oxydation mais ne pouvant directement être traités par les méthodes biologiques traditionnelles.

L'oxydation en milieu supercritique $\left(400^{\circ} \mathrm{C}<\mathrm{T}<600^{\circ} \mathrm{C}, 24\right.$ $\mathrm{MPa}<\mathrm{P}<40 \mathrm{MPa}$ ) est plus « poussée » pour des temps de séjour cent fois plus faibles grâce à l'élévation de température mais aussi grâce au fait que l'oxygène est totalement dissous dans ces conditions de pression. Un travail important de recherche fondamentale sur l'oxydation en milieux aqueux supercritiques ${ }^{[14-19]}$ a été initié dans les années 80 aux États Unis. Les chercheurs et industriels américains travaillent maintenant sur la faisabilité technique et industrielle de ce type de procédé. Le premier workshop sur l'oxydation en eau supercritique, qui s'est tenu à Jacksonville (Floride) en février 1995, permet de mesurer l'importance du travail 
effectué ${ }^{[20]}$. Plusieurs concepts de réacteurs ont été développés :

- réacteur tubulaire : un pilote est opérationnel depuis 1994 à Austin (Texas); il traite en continu $1000 \mathrm{l} / \mathrm{h}$ d'effluents liquides contenant des déchets organiques de l'industrie chimique ${ }^{[2]]}$;

- réacteur à paroi interne, poreuse ou à écoulement interne coaxial, qui permet de protéger de la corrosion les parois de l'enceinte haute pression ${ }^{[22,23]}$;

- réacteur réservoir à gradient de température qui consiste à injecter les effluents à basse température en partie haute du réacteur (Modar) ${ }^{[24,25]}$;

- réacteur multi-étapes, qui permet de gérer deux étapes (sous et supercritique) afin d'assurer l'avancement de la réaction de manière complémentaire ${ }^{[26,27]}$.

Enfin, il faut signaler qu'un brevet français propose de travailler en milieu réducteur permettant ainsi de générer des hydrocarbures légers et non plus du $\mathrm{CO}_{2}{ }^{[28]}$.

Au niveau européen, cinq laboratoires publics disposent de pilotes continus opérationnels de traitement d'effluents organiques et minêraux ( $I$ à $25 \quad l /$ heure) : le Forschungszentrum de Karlsruhe (Allemagne) ${ }^{[29]}$, I'Université de Valladolid (Espagne $)^{[30]}$, le CEA-Pierrelatte (France) $)^{[3]}$, l'Institut de chimie de la matière condensée de Bordeaux (France) ${ }^{[32]}$, l'Institut of Process Engineering and Cryogenics (Suisse) ${ }^{[33]}$.

Les études portent essentiellement sur la validation industrielle du procédé dans le cadre d'applications très spécifiques, l'inhibition des phénomènes de corrosion, la conception de nouveaux réacteurs.

Ainsi, bien que ces procédés nécessitent encore des études de base et technologiques, le fonctionnement du premier pilote de taille industrielle permet d'évaluer des coûts d'investissement et de fonctionnement équivalents à ceux d'une installation de traitement par incinération pour des boues contenant $10 \%$ de composés « organiques », avec des performances supérieures vis-à-vis de la protection de l'environnement. Les domaines d'applications propres à ces types de procédés concernent à titre d'exemple les molécules organiques difficilement biodégradables (pesticides,...) et les effluents liquides trop dilués pour être incinérés (purification des eaux industrielles ou domestiques,...).

\section{TRAVAUX RÉALISÉS A L'ICMCB}

Un de nos objectifs de travail sur le traitement hydrothermal de déchets industriels est de valider les potentialités technico-économiques de ce type de procédé. Dans ce contexte, nous nous intéressons aux mécanismes réactionnels et aux produits intermédiaires et finaux formés lors de l'oxydation ou de la réduction de l'effluent traité. Ces travaux étant réalisés sur des effluents industriels et fai- sant l'objet d'un dépôt de brevet ${ }^{[28]}$, nous nous limiterons à présenter dans cet article, pour des raisons de confidentialité, les études réalisées en oxydation sur des molécules modèles.

\section{Pilote expérimental}

La figure 2 présente une vue d'ensemble du dispositif expérimental de traitement des effluents liquides, que nous avons conçu et développé à l'ICMCB.

Les effluents aqueux (charge à traiter soluble) et les additifs nécessaires à la dissolution réactive des déchets sont introduits dans l'installation à la pression désirée par compression en phase liquide (pompe Lewa $50 \mathrm{MPa}$ ). Les effluents aqueux contenant des particules en suspension sont introduits par un circuit spécifique de chargement basse pression. La capacité maximale de traitement en effluent est de $3 \mathrm{~kg} / \mathrm{h}$ à $50 \mathrm{MPa}$.

Les effluents à traiter et l'additif sont ensuite préchauffés à la température désirée (système résistif ; température maximale de $400^{\circ} \mathrm{C}$ ) avant d'être mélangés à l'entrée du réacteur tubulaire en Inconel 718. La température de réaction est ensuite ajustée et régulée à la consigne de travail dans un four à bain de sable fluidisé chauffé par un système résistif (maximum $600^{\circ} \mathrm{C}$ ). La dissolution réactive peut donc être conduite en milieu oxydant ou réducteur dans des conditions souscritiques ou supercritiques.

Après réaction, les produits sont ensuite refroidis entre 20 et $30^{\circ} \mathrm{C}$ dans un échangeur à eau réfrigérée, puis détendus entre 0,1 et 0,5 MPa par une vanne Tescom de type déverseur.

Le séparateur gaz- liquide, couplé à une analyse chromatographique en phase gazeuse en ligne pour les effluents gazeux, permet de suivre en " temps réel », l'influence des para- 
mètres opératoires du pilote (pression, température, $\mathrm{pH}$, débit et concentration en additif principalement) sur la nature et la quantité de produits issus de la réaction. La phase liquide est caractérisée par chromatographie en phase gazeuse et par mesure de la demande chimique en oxygène (DCO) résiduelle.

Le contrôle commande du banc de traitement est réalisé sous l'architecture de l'ordinateur par le logiciel Labview (National Instruments).

\section{Résultats et discussions}

\section{Le méthanol}

Le méthanol a été choisi comme molécule modèle en raison de son caractère réfractaire à l'oxydation. Le schéma réactionnel global de la réaction d'oxydation du méthanol par le peroxyde d'hydrogène est le suivant :

$\mathrm{CH}_{3} \mathrm{OH}+3 \mathrm{H}_{2} \mathrm{O}_{2} \leftrightarrow \mathrm{CO}_{2}+5 \mathrm{H}_{2} \mathrm{O}$

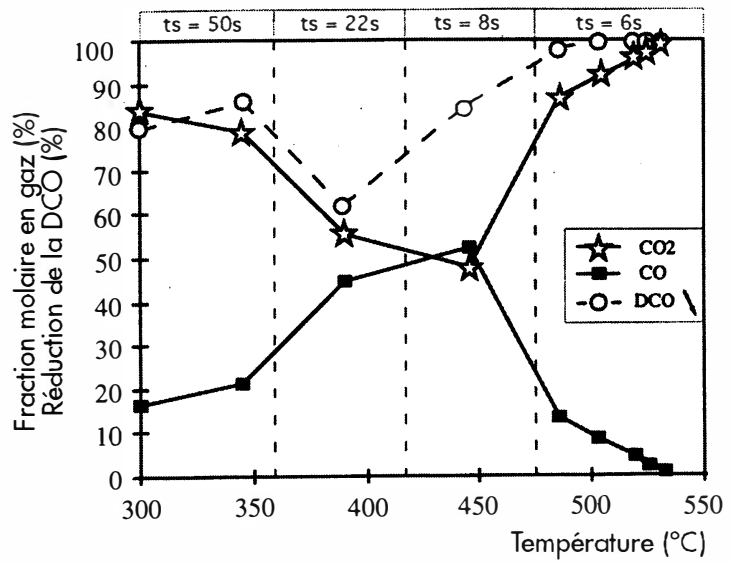

Figure 3 : Évolution de la fraction molaire en $\mathrm{CO} / \mathrm{CO}_{2}$ et de l'abattement de la $\mathrm{DCO}$ en $\%$ en fonction de la température de la réaction d'oxydation du méthanol par le peroxyde d'hydrogène à $25 \mathrm{MPa}$

La figure 3 montre l'évolution de l'abattement de la DCO en fonction de la température de traitement. Cet abattement permet d'obtenir une DCO résiduelle dans l'effluent liquide, inférieure à la norme actuelle de rejet pour une température de $510^{\circ} \mathrm{C}$, alors que la composition de l'effluent gazeux montre un pourcentage de $\mathrm{CO}$ supérieur à $5 \%$. II faut atteindre une température de $530^{\circ} \mathrm{C}$ et un temps de séjour de $6 \mathrm{~s}$ pour avoir un abattement de la DCO supérieur à $99,9 \%$ et une production de $\mathrm{CO}_{2}$ suivant l'équation I supérieure à $99,5 \%$. A $400^{\circ} \mathrm{C}$, nous pouvons noter une diminution de l'abattement de la DCO alors que la température de réaction croît. Ceci est directement relié à la diminution du temps de séjour dans le réacteur induite par la diminution de masse volumique du fluide avec l'augmentation de la température à pression constante (figure I). En effet, nous travaillons à débit massique constant et une diminution de la masse volumique conduit à une augmentation de la vitesse dans le réacteur.

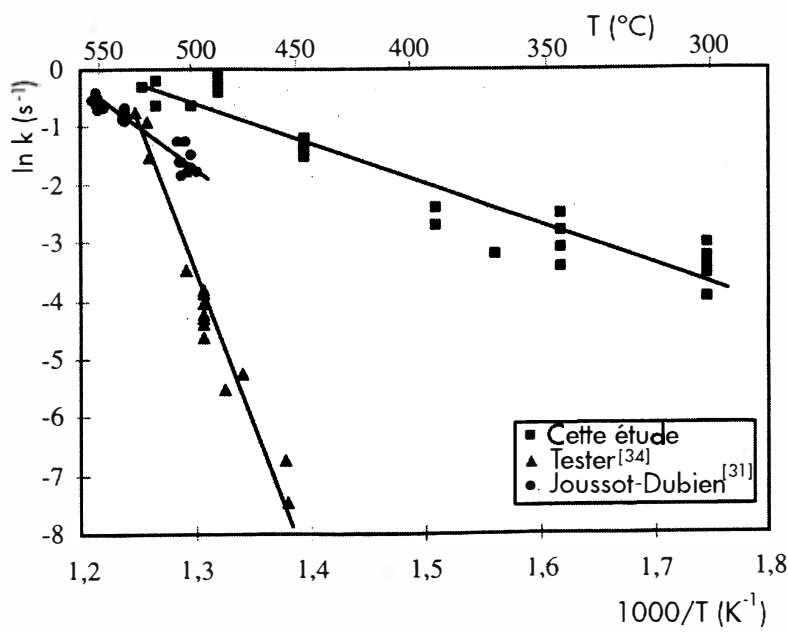

Figure 4 : Évolution de la constante globale de réaction d'oxydation du méthanol en fonction de la température à $25 \mathrm{MPa}$

Nous avons réalisé l'étude cinétique des réactions avec un formalisme du type loi d'Arrhénius où la constante de réaction s'exprime par la relation suivante :

$k=A \exp (-E a / R T)$

où Ea représente l'énergie d'activation et $A$ le facteur préexponentiel.

Pour la réaction d'oxydation du méthanol par le peroxyde d'hydrogène (figure 4) en supposant une réaction d'ordre I, nous avons obtenu une valeur de $58 \pm 10 \mathrm{~kJ} /$ mole pour Ea et de $4800 \mathrm{~s}^{-1}$ pour $A$. Ces résultats sont à comparer avec ceux de Tester $\left(E a=409 \mathrm{~kJ} / \mathrm{mole}, A=1026,5 \mathrm{~s}^{-1}\right)^{[34]}$ et ceux de Joussot-Dubien $\left(E a=115 \mathrm{~kJ} / \mathrm{mole}, A=107 \mathrm{~s}^{-1}\right)^{[31]}$ qui sont reportés sur la figure 4 . Les différences entre nos travaux et ceux de Tester sont liés au fait que Tester utilise de l'oxygène comme oxydant qui est "réputé » être moins réactif que le peroxyde d'hydrogène. En effet, le peroxyde d'hydrogène génère des radicaux $\mathrm{OH}$ qui conduisent à des composés intermédiaires plus réactifs que ceux formés en présence d'oxygène ${ }^{[35]}$. Nos travaux confirment bien ces. résultats lorsque nous les comparons à ceux obtenus par Joussot-Dubien qui préchauffe l'eau oxygénée à $400^{\circ} \mathrm{C}$ avant de l'injecter dans le réacteur de traitement. Dans ces conditions expérimentales, le peroxyde d'hydrogène a déjà commencé à se décomposer en $\mathrm{O}_{2}$ et $\mathrm{H}_{2} \mathrm{O}^{[35]}$. Joussot-Dubien obtient ainsi, comme le montre la figure 4 , des résultats intermédiaires entre ceux de Tester et ceux de notre étude. Nous avons pu ainsi définir, avec le méthanol comme molécule modèle, les conditions de fonctionnement opératoires les plus contraignantes du point de vue de l'abattement de la $\mathrm{DCO}$ et des produits liquides et gazeux en sortie de l'installation.

\section{Le glucose}

Le glucose a été choisi comme molécule modèle en raison de son caractère générique en oxydation de déchets cellulosiques et plus généralement du traitement de la biomas- 


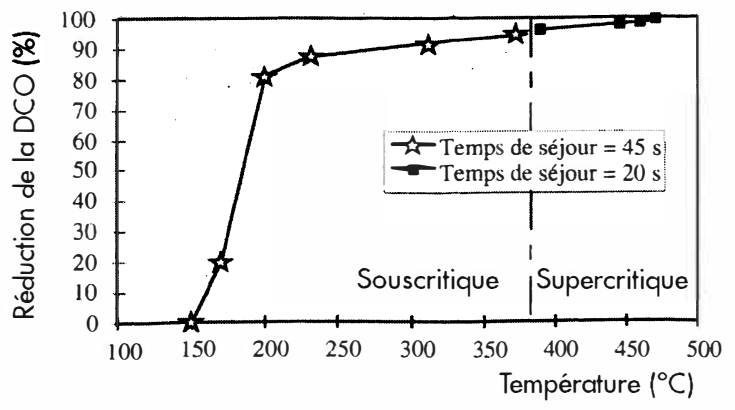

Figure 5 : Évolution de l'abattement de la DCO en \% en fonction de la température de la réaction d'oxydation du glucose par le peroxyde d'hydrogène à $\mathbf{2 5}$ $\mathrm{MPa}$

se. Le schéma réactionnel global de la réaction d'oxydation du glucose par l'eau oxygénée est le suivant :

$\mathrm{C}_{6} \mathrm{H}_{12} \mathrm{O}_{6}+12 \mathrm{H}_{2} \mathrm{O}_{2} \leftrightarrow 6 \mathrm{CO}_{2}+18 \mathrm{H}_{2} \mathrm{O}$

(I)

La figure 5 montre l'évolution de l'abattement de la DCO en fonction de la température de traitement. L'abattement de DCO est de $99,9 \%$ pour une température de $470^{\circ} \mathrm{C}$ avec des effluents gazeux composés à $99,5 \%$ de $\mathrm{CO}_{2}$. II est intéressant de noter que des températures inférieures, même si le temps de séjour est doublé, ne permettent pas un abattement suffisant de la DCO. Antal ${ }^{[36]}$, sur le traitement pyrolytique du glucose en milieu aqueux à $600^{\circ} \mathrm{C}$ et $34 \mathrm{MPa}$, obtient une gazéification complète pour des concentrations inférieures à $0, I \mathrm{~mol} / \mathrm{l}$. L'utilisation d'un additif oxydant permet donc d'abaisser fortement la température et la pression de travail.

Le traitement du glucose nous a ainsi permis de définir, à titre d'exemple, les conditions opératoires de traitement hydrothermal des boues de désencrage de pâtes à papier.

\section{CONCLUSION}

Les résultats obtenus à l'ICMCB sur le traitement de molécules modèles, représentatives d'une part des molécules organiques réfractaires à l'oxydation et d'autre part des dérivés cellulosiques, confirment clairement les performances du traitement hydrothermal des déchets du point de vue de leur gestion dans un cadre législatif toujours plus contraignant. Dans une optique de validation industrielle, il est nécessaire d'identifier les composés intermédiaires et finaux et de maîtriser l'évolution des sous produits rejetés en fonction des conditions opératoires (DCO, COT, MES,...). L'optimisation du procédé quant à elle, implique la connaissance des mécanismes réactionnels mis en jeu et des bilans énergétiques.

Remerciements : Nous remercions l'Agence de l'environnement et de la maitrise de l'énergie (Ademe) et la région Aquitaine pour leurs soutiens financiers.

* P. Beslin, F. Cansell, Y. Garrabos, G. Demazeau, ICMCB, CNRS, Université Bordeaux I, 33608 Pessac cedex - France

\section{** B. Berdeu, D. Sentagnes,}

L'Électrolyse, Zone Industrielle, 33360 Latresne - France

\section{Bibliographie}

[I] E. Schmidt, Properties of water and Rapport CEA-CNRS (Feb. 1995) steam in S.I. units, Third, Enlarged printing, Ed Grigull U. (1982)

[2] K. Heger, M. Uematsu, E.U. Franck, Ber. Bunsenges. Phys. Chem., 84, 758 (1980)

[3] E.U. Franck, J. Chem. Thermodynamics, 19, 225 (1987)

[4] G. Antonini, P. Gislais, Traitements thermiques des déchets industriels, Ed Lavoisier, 4I (1995)

[21] W.O. 95/26929, 12.10.95, Eco Waste Technologies, Method and apparatus for treating waste water streams

[22] W.O. 94/18128, 18.08.1994, Mc Guiness T.G. ; Supercritical oxidation reactor appartus and method

[23] U.S. 5387398, 07.02.1995, Aerojet General Corporation ; Supercritical water oxidation reactor with wall condsuits for boundary flow control

[5] J. Besombes-Vailhé, H. [24] U.S. 523260, 03.08.1993, Modar Debellefontaine, J.N. Foussard, J. Env. Engin., 115, 367 (1989)

[6] A.B. Bjerre, E. Rasmussen, E. Serensen, Env. Techn., II, 429 (1990) [7] A.M. Balinsky, D.P. Harisson, D.D. Reible, D.M. Wetzel, R.S. Willms, Env progress, 4, I3। (1985)

[8] U.S. 4350599, 21.09.1982 Sterling Drug; Process for treatment of caustic water liquors

[9] U.S. 4812243, 14.03.1989, Zimpro: Process for treating caustic cyanide metal wastes

[10] C.H. 681359, 15.03.93, Ciba Geigy; Reaktor fur nassoxidation

[I I] U.S. 4803054, 07.02.89, Vertech Treatment Systems, Inc. ; Asymmetric heat exchange reaction apparatus for effecting chemical reactions

[12] Fr. 94 05005, 26.04.1994, CEA CEERTE ; Installation pour le traitement de déchets hétérogènes par oxydation en voie humide

[13] E. Pongratz, S. Peter, G. Hartel, C. Freese, High Pressure Chemical Engineering, Ed. Ph. Rudolf von Rohr, Ch. Trepp, Process Technology Proceeding, 12, 55 (1996) Inc. ; Process for oxidation of materials in water at supercritical temperature utilizing reaction rate enchancers

[25] U.S. 5358645, 25.10.1994, Modar Inc. ; Zirconium oxide ceramics for surfaces exposed to high temperature water oxidation environments

[26] U.S. 5358646, 25.10.1994, Gloyna E.F., Li L., Mc Brayer R.N. ; Method and apparatus for multi-stage and recycle wet oxidation

[27] U.S. 5240619, 31.08.1993, Zimpro Passavant Environmental. Two stage subcritical-supercritical wet oxidation

[28] Fr. 94 14664, 06.12.1994, L'Électrolyse ; Procédé en milieu réducteur de transformation chimique de structures chimiques complexes dans un fluide supercritique

[29] H. Goldacker, J. Abeln, M. Kluth, A. Kruse, H ; Schmieder, G. Wiegand, High Pressure Chemical Engineering, Ed. Ph. Rudolf von Rohr, Ch. Trepp, Process Technology Proceeding, 12, 61 (1996)

[30] M.]. Cocero, J.L. Soria, O. Ganado, R. Gonzalez, F. Fdez-polanco, High Pressure Chemical Engineering, Ed. Ph. Rudolf von Rohr, Ch. Trepp, Process

[14] E. E. Brock, Ph. E. Savage, AlChE Technology Proceeding, 12, I2I (1996) Journal, 4I, 1874 (1995)

[31] C. Joussot-Dubien, Thèse [15] P. Dagaut, B. Daney de Marcillac, Université Bordeaux I (1996)

Y. Tan, M. Cathonnet, J.C. Boettner, J Chim. Phys., 92, 1124 (1995)

[16] S. Gopalan, Ph. E. Savage, AIChE Journal, 4I, 1864 (1995)

[17] Ph. E. Savage, S. Gopalan, T. I. Mizan, C. J. Martino, E. E. Brock, AIChE Journal, 4I, 1723 (1995)

[18] Luck F., Bonnin C., Niel G., Naud G., First international workshop on supercritical water oxidation, Jacksonville-USA (Feb. 1995)

[19] Bouki S.N., Landvatter R., Habicht W., Fraz G., Leishkow S., Kraft R., Jacobi O., First international workshop on supercritical water oxidation. Jacksonville-USA (Feb. 1995)

[20] C. Joussot-Dubien, F. Cansell,
[32] P. Beslin, A. Largeteau, G. Demazeau, Y. Garrabos, F. Cansell, D. Sentagnes, B. Berdeu, Récents progrès en génie des procédés, 9 (43), 49 (1995)

[33] H.L.La Roche, M. Weber, Ch. Trepp, High Pressure Chemical Engineering, Ed. Ph. Rudolf von Rohr, Ch. Trepp, Process Technology Proceeding, 12, 645 (1996)

[34] J.W.Tester, P.A. Webley, H.R. Holgate, Ind. Eng. Chem. Res., 32, 236 (1993)

[35] D. Lee, E.F. Gloyna, L. Lixiong, J. Supercrit. Fluids, 3, 249 (1990)

[36] M.J. Antal, Phys. Chem. Aqueous Syst., Eds White, J. Howard, 24 (1995) 